\title{
ĐÁNH GIÁ Độ ỔN ĐỊNH CỦA VIÊN NANG VERAPAMIL HYDROCLORID 120MG GIẢI PHÓNG KÉO DÀI
}

\section{TÓM TẮT.}

Mục tiêu: Đánh giá được độ ổn định của viên nang Verapamil hydroclorid (VER.HCl) $120 \mathrm{mg}$ giải phóng kéo dài ở điêu kiện thực và điều kiện lão hóa cấp tốc theo qui định của FDA và Asean. Phương pháp: Định lượng VER.HCl bằng phương pháp quang phổ hấp thụ tử ngoại và HPLC; đánh giá độ hòa tan của viên nang VER.HCl $120 \mathrm{mg}$ giải phóng kéo dài theo phương pháp của USP 41; đánh giá độ ổn định của viên nang VER.HCl $120 \mathrm{mg}$ giải phóng kéo dài ở điều kiên thực và điều kiện lão hóa cấp tốc (nhiệt đô $40 \pm 1^{\circ} \mathrm{C}$, độ ẩm $75 \%$ ); các chỉ tiêu đánh giá gồm: hình thức viên, hàm lượng VER. $\mathrm{HCl}$ và độ hòa tan. Kết quả: Trong 18 tháng bảo quản ở điêu kiện thực và 6 tháng bảo quản ở điều kiện lão hóa cấp tốc, các chỉ tiêu chất lượng (hình thức, độ hòa tan, hàm lượng) của viên nang VER.HCl $120 \mathrm{mg}$ giải phóng kéo dài hầu như không thay đổi. Kết luận: Sau khi bảo quản 18 tháng ở điêu kiện thực và 6 tháng ở điều kiện lão hóa cấp tốc, các chỉ tiêu chất lượng của viên viên nang VER.HCl $120 \mathrm{mg}$ giải phóng kéo dài thay đổi không đáng kể và đạt yêu cầu tiêu chuẩn cơ sở của chế phẩm.

Tứ khóa: Verapamil hydroclorid, giải phóng kéo dài, độ ổn định.

\section{SUMMARY}

\section{EVALUATION OF STABILITY OF VERAPAMIL HYDROCHLORIDE 120 MG SUSTAINED RELEASE CAPSULES}

Objectives: To evaluate the stability of Verapamil hydrochloride (VER. $\mathrm{HCl}) 120 \mathrm{mg}$ sustained release capsules using real-time stability testing and accelerated aging stability testing according to FDA and Asean guidelines. Methods: Quantification of VER. HCl by ultraviolet absorption spectroscopy and HPLC; evaluate the solubility of VER.HCl $120 \mathrm{mg}$ sustained-release capsules according to the method in USP 41; evaluate the stability of VER.HCl $120 \mathrm{mg}$ sustained-release capsules under real condition and accelerated aging condition (temperature $40 \pm 1^{\circ} \mathrm{C}$, humidity $75 \%$ ); The evaluation parameters included: pellet appearance, VER.HCl content and solubility. Results: During 18 months of storage in real condition and 6 months of storage in accelerated aging condition, the quality parameters (appearance, solubility, content) of the VER. $\mathrm{HCl} 120 \mathrm{mg}$ sustainedrelease capsules were almost constant. Conclusion:

*Học viện Quân y

Chịu trách nhiệm chính: Trương Đức Mạnh,

Email: manhxn150@gmail.com

Ngày nhận bài: 28/8/2021

Ngày phản biện khoa học: 25/9/2021

Ngày duyệt bài: 1/11/2021

\author{
Trương Đức Mạnh1, Võ Xuân Minh², \\ Phan Thị Hòa ${ }^{1}$, Nguyễn Văn Bạch ${ }^{1}$
}

After 18 months of storage in real condition and 6 months under accelerated aging condition, the quality parameters of extended-release VER. HCl $120 \mathrm{mg}$ capsules were stable and passed in-house specification of product.

Keywords: Verapamil hydrochloride, sustained release, stability

\section{I. ĐăT VẤN ĐỀ}

Verapamil hydroclorid (VER.HCl) là một dược chất có tác dụng chẹn kênh calci, được dùng để điều trị đau thắt ngực, tăng huyết áp và rối loạn nhịp tim. VER.HCl được hấp thu hoàn toàn qua đường tiêu hóa (khoảng 90\%), tuy nhiên sinh khả dụng chỉ đạt 20 - 35\% do chuyển hóa bước đầu qua gan nhanh. Dược chất có thời gian bán thải ngắn (2,8-7,4 giờ) khiến cho bệnh nhân phải uống thuốc nhiêu lân trong ngày [1], [2], [3]. Do đó việc bào chế VER.HCl dưới dạng viên nang giải phóng kéo dài rất có ý nghĩa trong điêuu trị. Đã nghiên cứu bào chế thành công viên nang VER.HCl 120 mg giải phóng kéo dài, chế phẩm đạt tiêu chuẩn cơ sở theo các tiêu chí về viên nang của DĐVN IV và USP 41 [4]. Do đó, tiến hành đánh giá độ ổn định của chế phẩm ở điều kiện thường và điều kiện lão hóa cấp tốc với mục đích chứng minh chế phẩm vẫn đạt các tiểu chuẩn sau hai điều kiện bảo quản.

\section{II. ĐỐI TƯỢNG VÀ PHƯƠNG PHÁP NGHIÊN CỨU \\ 2.1. Nguyên vật liệu}

- Verapamil hydroclorid chuẩn: SKS QT242010914, hàm lượng $100,52 \%$ do Viện kiểm nghiệm thuốc trung ương cung cấp.

- Viên nang VER.HCl 120 mg giải phóng kéo dài đạt tiêu chuẩn cơ sở.

- Methanol, acetonitril, Kali dihydrophosphat: Đạt tiêu chuẩn P.A (Merck Đức).

\subsection{Thiết bị nghiên cứu}

- Máy thử độ hoà tan Copley DIS 8000 (Anh).

- Máy đo quang phổ UV - VIS Labomed UVD2960 (Mỹ).

- Hệ thống HPLC Alliance Waters 2695D; cột

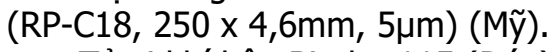

- Tủ vi khí hậu Binder 115 (Đức).

- Cân phân tích Mettler toledo có độ chính xác 0,1mg (Thụy Sỹ).

- Màng lọc nilon Sartorius Minisart kích thước

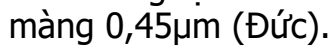


- Hê thống phễu thuỷ tinh Sartotius (Đức).

- Các dụng cụ khác đạt tiêu chuẩn phân tích.

\subsection{Phương pháp nghiên cứu}

\subsubsection{Phương pháp đánh giá đô ổn đinhh} Được thực hiện theo quy định của FDA [5] và Asean [6].

Đối tượng thử: Các mẫu viên nang VER.HCI $120 \mathrm{mg}$ giải phóng kéo dài của 3 lô khác nhau (mỗi lô khoảng 1000 viên) được ép vỉ PVC/nhôm 10 viên, đóng trong hộp carton, mồi hộp 2 vỉ.

*Điếu kiện và thời gian bảo quản:

- Nghiên cứu dài hạn: Điều kiện thực tại phòng thí nghiệm, thời gian bảo quản 18 tháng.

- Nghiên cứu lão hoá cấp tốc:

+ Nhiêt độ: $40 \pm 2^{\circ} \mathrm{C}$.

+ Độ ẩm: $75 \pm 5 \%$.

+ Thời gian bảo guản: 6 tháng.

*Thời gian lấy mâu: Sau khi bảo quản 1, 3, 6 , 9,12 và 18 tháng (đối với điều kiện thường) và 1,3 và 6 tháng (đối với điều kiện lão hoá cấp tốc).

*Các tiêu chuẩn khảo sát:

Đánh giá các chỉ tiêu: Hình thức, hàm lượng VER.HCl và độ hoà tan.

2.3.2. Phương pháp đánh giá tiêu chuẩn chất lượng

*Hình thức viên: Viên nang cứng số 1 , thân màu trắng, nắp màu đỏ. Bên trong chứa các hạt pellet nhỏ, hình cầu, màu trắng.

*Định lượng VER. $\mathrm{HCl}$ trong viên nang bằng phương pháp HPLC:

- Mấu chuẩn: Cân chính xác khoảng $20 \mathrm{mg}$ VER. $\mathrm{HCl}$ vào bình định mức $100 \mathrm{ml}$, hòa tan vừa đủ $100 \mathrm{ml}$ bằng methanol được dung dịch $\mathrm{A}$. Lây chính xác $5,0 \mathrm{ml}$ dung dịch $\mathrm{A}$ cho vào bình định mức $50 \mathrm{ml}$, pha loãng vừa đủ $50 \mathrm{ml}$ bằng methanol, lọc qua màng lọc đường kính $0,45 \mu \mathrm{m}$ được mẫu chuẩn có nồng độ khoảng $20 \mu \mathrm{g} / \mathrm{ml}$.

- Mẫu thử: Cân 20 viên nang, xác đinh khối lượng trung bình bột thuốc trong nang và nghiền thành bột minn, trộn đều, đo hàm ẩm của bột. Cân một lượng bột viên tương đương $20 \mathrm{mg}$ VER.HCl cho vào bình định mức $100 \mathrm{ml}$, thêm khoảng $50 \mathrm{ml}$ methanol, lắc siêu âm 30 phút ở $30^{\circ} \mathrm{C}$ trong 15 phút, sau đó để ổn định về nhiệt độ phòng, thêm methanol vừa đủ $100 \mathrm{ml}$, lọc qua giấy lọc (bỏ 20ml dịch lọc đầu) được dung dịch $\mathrm{B}$. Lây chính xác $5,0 \mathrm{ml}$ dung dịch $\mathrm{B}$ cho vào bình định mức $50 \mathrm{ml}$, pha loãng vừa đủ $50 \mathrm{ml}$ bằng methanol, lọc qua màng lọc đường kính $0,45 \mu \mathrm{m}$ được mẫu thử.

- Thiết bị và điều kiên sắc ký:

+ Hê thổng HPLC Alliance Waters 2695D.

+ Pha tĩnh: Cột SunFireTM (RP-C18, $250 \times 4,6 \mathrm{~mm}, 5 \mu \mathrm{m}$ )
+ Nhiệt độ cột: $25-26^{\circ} \mathrm{C}$.

+ Detector: UV tại bước sóng 278 nm

+ Pha động: Acetonitril:Đệm $\mathrm{KH}_{2} \mathrm{PO}_{4}$ 0,05M

$\mathrm{pH} 3,6(40: 60)$

+ Thể tích tiêm: $20 \mu \mathrm{l}$.

+ Tốc độ dòng: 1,0 ml/phút.

- Công thức tính:

HL $(\%)=\frac{\text { St } \times \mathrm{Cs} \times \mathbf{m}}{120 \times \mathrm{Ss} \times \mathrm{mc} \times(100-\mathbf{a})} \times 10000$

Trong đó:- St: Diện tích pic của mẫu thử $(\mu \mathrm{V} . \mathrm{s})$

- Cs: Nồng độ thực tế của mẫu chuẩn $(\mu \mathrm{g} / \mathrm{ml})$

- Ss: Diện tích pic của mẫu chuẩn ( $\mu \mathrm{V} . \mathrm{s})$

- m: Khối lượng trung bình của một viên chế phẩm $(\mathrm{mg})$

- mc: Khối lượng bột viên (mg)

- a: Hàm ẩm của bột viên (\%)

*Định lượng VER. $\mathrm{HCl}$ trong môi trường hòa tan bằng phương pháp quang phổ UV-Vis

- Pha dung dịch VER.HCl chuẩn có nồng độ khoảng $50 \mu \mathrm{g} / \mathrm{ml}$ trong môi trường đệm phosphat $\mathrm{pH} 7,5$. Tiến hành ghi phổ trong vù̀ng có bước sóng từ $200-400 \mathrm{~nm}$. Sau đó, so sánh với phô̂ của dung dịch VER. $\mathrm{HCl}$ chuẩn với phổ của dung dịch hỗn hợp tá dược để kiểm tra tại $\lambda_{\text {max }}$ của VER.HCl.

- Xây dựng đường chuẩn: Pha dung dịch VER.HCl chuẩn trong môi truờng đệm phosphat $\mathrm{pH} 7,5$ có nồng độ là: $30,40,50,60$ và 70 $\mu \mathrm{g} / \mathrm{ml}$. Đo mật độ quang của các dung dịch trên tai $\lambda_{\max }$ của VER. $\mathrm{HCl}$ với mẫu trắng là dung dịch đềm phosphat $\mathrm{pH} 7,5$. Đánh giá đường chuẩn dựa vào hệ số tương quan và đánh giá độ lặp lại của phương pháp dựa vào giá trị RSD.

- Nồng độ của dung dịch VER.HCl thử được tính theo công thức sau:

Trong đó:

$$
C_{x}=\frac{C_{a} \times E_{x}}{\mathbf{E}_{a}}
$$

- $C_{x}$, $C_{a}$ : Nồng độ dung dịch VER.HCl chuẩn và dung dịch thử $(\mu \mathrm{g} / \mathrm{ml})$

- $E_{a}, E_{x}$ : Độ hấp thụ của dung dịch VER.HCl chuẩn và dung dịch thử.

(Ghi chú: Nồng độ của VER. $\mathrm{HCl}$ chuẩn và thử phải nằm trong khoảng nồng độ chuẩn 30-70 $\mu \mathrm{g} / \mathrm{ml}$. Nếu mật Đố hấp thụ lớn thì phải pha loãng và nếu Độ hấp thụ bé thì sử dụng phương pháp thêm chuẩn).

*Phương pháp đánh giá độ hòa tan của pellet VER.HCl $120 \mathrm{mg}$ giải phóng kéo dài: theo chuyên luận "Verapamil Hydrochloride Extended-Release Tablets, Test 5" USP 41 với các điều kiện cụ thể sau:

- Thiết bị: cánh khuấy.

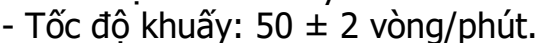


- Nhiệt độ: $37,0 \pm 0,5^{\circ} \mathrm{C}$.

- Môi trường: 900ml dung dịch đệm phosphat $\mathrm{pH} 7,5$.

- Thời gian lấy mẫu: $1,2,4$ và 8 giờ.

- Mẫu thử: viên nang VER.HCl 120 mg giải phóng kéo dài.

- Định lượng DC giải phóng ở các thời điểm bằng phương pháp đo độ hấp thụ ở bước sóng $\lambda_{\max }$. Tính lượng giải phóng $\mathrm{DC}$ căn cứ vào đường chuẩn của VER. $\mathrm{HCl}$ trong môi trường đệm phosphat $\mathrm{pH} 7,5$ bằng phương pháp quang phổ UV-VIS [7].

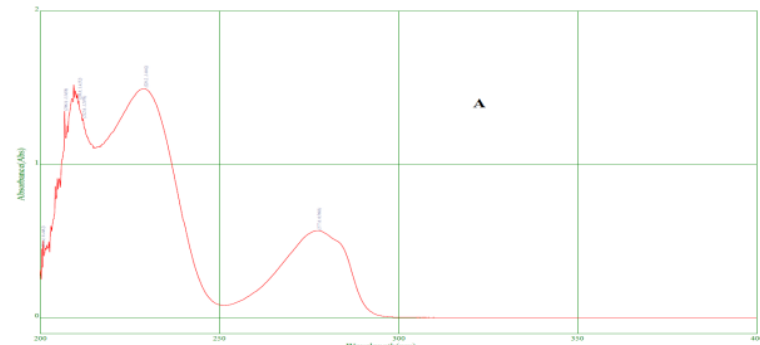

\section{KẾT QUẢ NGHIÊN CỨU VÀ BÀN LUÂ̂N}

3.1. Kết quả xây dựng phương pháp định lượng VER.HCI bằng quang phổ UV-Vis

Pha dung dịch VER.HCl chuẩn có nồng độ khoảng $50 \mu \mathrm{g} / \mathrm{ml}$ trong môi trường đệm phosphat $\mathrm{pH} 7,5$. Tiến hành ghi phổ trong vùng có bước sóng từ 200-400 nm. Sau đó, so sánh với phổ của dung dịch chứa VER. $\mathrm{HCl}$ và phổ của dung dịch hỗn hợp tá dược trong pellet. Kết quả được trình bày ở hình 1.

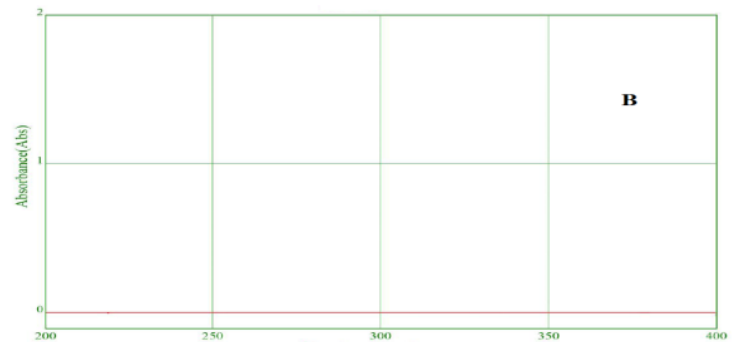

Hình 1. Phổ UV-Vis của dung dich VER.HCl (a) và

hỗn hợp tá dược (b) trong dung dịch đệm phosphat pH 7,5

Kết quả cho thấy: Trong khoảng từ 200400nm, dung dịch VER.HCl có cực đại hấp thụ tại $278 \mathrm{~nm}$ và tại bước sóng này, phổ của dung dịch tá dược khổng xuất hiện đỉnh hấp thụ. Điều đó chứng tỏ tá dược không ảnh hưởng đển phép đo quang phổ của VER.HCl. Do đó, có thể dùng phương pháp đo quang phổ hấp thụ UV tại bước sóng $278 \mathrm{~nm}$ để xác định nồng độ VER.HCl trong quá trình đo độ hoà tan.

Xây dựng đường chuẩn: Pha dung dịch VER. $\mathrm{HCl}$ trong dung dịch đệm phosphat $\mathrm{pH} 7,5$ có các nồng độ lần lượt là: $30,40,50,60$, và $70 \mu \mathrm{g} / \mathrm{ml}$. Đo độ hấp thụ tại bước sóng $278 \mathrm{~nm}$. Vẽ đồ thị tương quan giữa độ hấp thụ và nồng độ VER. HCl. Kết quả được trình bày ở bảng 1 và hình 2.

Bảng 1. Kêt quả đo độ hâp thụ của dung dịch VER.HCl trong đệm phosphat pH 7,5 $(n=5)$

\begin{tabular}{|c|c|c|c|c|c|}
\hline Nộ hấp thụ & $\mathbf{3 0}$ & $\mathbf{4 0}$ & $\mathbf{5 0}$ & $\mathbf{6 0}$ & $\mathbf{7 0}$ \\
\hline$\overline{\mathbf{A}}$ & 0,2558 & 0,3619 & 0,4589 & 0,5676 & 0,7064 \\
\hline RSD (\%) $(\boldsymbol{\%} \mathbf{g} / \mathbf{m l})$ & 3,37 & 2,83 & 0,70 & 1,28 & 0,26 \\
\hline
\end{tabular}

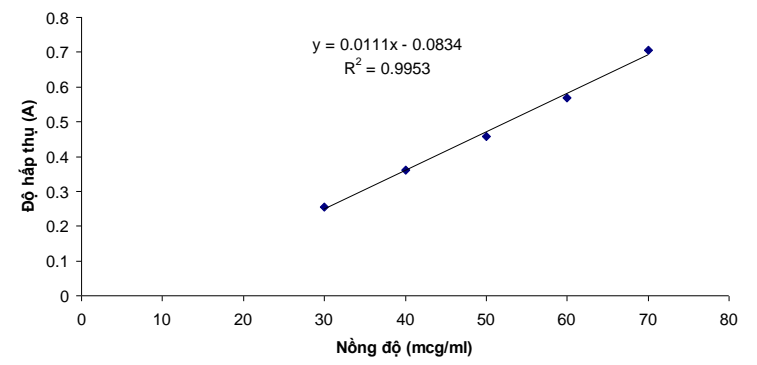

Hình 2. Đường chuẩn của dung dịch VER.HCI trong môi trường đệm phosphat $\mathrm{pH} 7,5$

Kết quả xây dựng đường chuẩn cho thấy: Trong khoảng nồng độ khảo sát từ $30-70 \mu \mathrm{g} / \mathrm{ml}$, đường chuẩn của VER. $\mathrm{HCl}$ thu được là một đường thẳng tuyến tính, có phương trình hồi quy $y=0,0111 x-0,0834$ với hệ số $R^{2}=0,9953$. Như vậy, có sự tương quan tuyến tính giữa mật độ quang và nồng độ dung dịch trong khoảng khảo sát. Do đó, có thể sử dụng phương pháp quang phổ hấp thụ UV tại bước sóng 278 nm để định lượng VER.HCl giải phóng trong quá trình hoà tan.

3.2. Kết quả đánh giá độ ổn định của viên nang VER.HCl $120 \mathrm{mg}$ giải phóng kéo dài

3.2.1. Về hình thức. Các viên nang VER. $\mathrm{HCl}$ $120 \mathrm{mg}$ giải phóng kéo dài bảo quản 18 tháng ở điều kiện thực và điều kiện lão hoá cấp tốc không có thay đổi so với thời điểm ban đâu.

3.2.2. Về hàm lượng VER.HCl trong viên nang VER.HCl $120 \mathrm{mg}$ giải phóng kéo dài. Sự thay đổi hàm lượng VER. $\mathrm{HCl}$ của chế phẩm trong quá trình bảo quản ở điều kiện thực (18 tháng) và điều kiện lão hoá cấp tốc (6 tháng) được trình bày ở bảng 2 và 3 . 
Bảng 2. Hàm lượng (\%) VER.HCl trong viên nang VER.HCl 120mg giải phóng kéo dài bảo quản ở điều kiện thực $(n=5 ; \bar{X} \pm S D)$

\begin{tabular}{|c|c|c|c|}
\hline Thời gian bảo quản (tháng) & Mẻ 1 & Mẻ 2 & Mẻ 3 \\
\hline 0 & $101,72 \pm 3,81$ & $101,43 \pm 3,89$ & $100,66 \pm 4,22$ \\
\hline 1 & $101,44 \pm 4,36$ & $100,85 \pm 4,24$ & $100,05 \pm 3,88$ \\
\hline 3 & $100,70 \pm 3,55$ & $99,85 \pm 3,58$ & $99,18 \pm 4,05$ \\
\hline 6 & $99,84 \pm 4,18$ & $99,36 \pm 3,94$ & $98,78 \pm 3,92$ \\
\hline 9 & $99,31 \pm 4,79$ & $98,94 \pm 4,15$ & $98,19 \pm 4,42$ \\
\hline 12 & $98,76 \pm 4,82$ & $98,37 \pm 4,64$ & $97,69 \pm 3,26$ \\
\hline 18 & $97,54 \pm 4,37$ & $97,12 \pm 4,02$ & $96,91 \pm 4,08$ \\
\hline
\end{tabular}

Bảng 3. Hàm lương (\%) VER.HCl trong viên nang VER.HCl 120mg giải phóng kéo dài bảo quản ở điều kiện lão hoá cấp tốc $\left(40 \pm 2^{\circ} \mathrm{C} ; 75 \pm 5 \% R H\right)(n=5 ; \bar{X} \pm S D)$

\begin{tabular}{|c|c|c|c|}
\hline Thời gian bảo quản (tháng) & Mẻ 1 & Mẻ 2 & Mẻ 3 \\
\hline 0 & $101,72 \pm 3,81$ & $101,43 \pm 3,89$ & $100,66 \pm 4,22$ \\
\hline 3 & $99,81 \pm 4,12$ & $99,10 \pm 3,73$ & $98,34 \pm 4,36$ \\
\hline 6 & $98,04 \pm 3,30$ & $97,46 \pm 3,77$ & $97,08 \pm 4,13$ \\
\hline
\end{tabular}

Nhận xét: Hàm lượng VER.HCl trong chế phẩm không giảm nhiều trong 18 tháng bảo quản ở điều kiện thường (giảm khoảng 3,754,31\%). Sau 6 tháng ở điều kiện lão hoá cấp tốc, hàm lượng VER.HCl giảm khoảng 3,58-3,97\%. Như vậy, hàm lượng VER.HCl trong các viên chỉ giảm nhẹ ở cả 3 lô bào chế. Tất cả các viên vẫn

đạt tiêu chuẩn về hàm lượng.

3.2.3. Về độ hoà tan. Kết quả đánh giá độ hòa tan của viên nang VER. HCl $120 \mathrm{mg}$ giải phóng kéo dài từ các viên ở 3 lô bào chế sau 18 tháng bảo quản ở điều kiện thực được trình bày ở bảng 4, 6 tháng bảo quản ở điều kiện lão hóa cấp tốc được trình bày ở bảng 5 .

Bảng 4. Độ hoà tan của viên nang VER.HCl 120mg giải phóng kéo dài bảo quản ở điều kiện thực $(n=6 ; X \pm S D)$

\begin{tabular}{|c|c|c|c|c|c|}
\hline \multirow{2}{*}{ Lô } & \multirow{2}{*}{ Tháng } & \multicolumn{3}{|c|}{ Tỷ lệ \% VER. HCl giải phóng theo thời gian (giờ) } \\
\cline { 2 - 6 } & & 1 & 2 & 4 & 8 \\
\hline \multirow{4}{*}{ I } & 0 & $7,96 \pm 0,19$ & $20,03 \pm 0,99$ & $43,39 \pm 1,61$ & $82,05 \pm 2,98$ \\
\cline { 2 - 6 } & 1 & $8,12 \pm 0,28$ & $20,15 \pm 1,00$ & $43,70 \pm 1,42$ & $83,57 \pm 3,43$ \\
\cline { 2 - 6 } & 3 & $8,06 \pm 0,30$ & $20,28 \pm 0,98$ & $43,21 \pm 2,09$ & $83,67 \pm 3,40$ \\
\cline { 2 - 6 } & 6 & $8,03 \pm 0,21$ & $20,75 \pm 0,99$ & $43,64 \pm 1,33$ & $82,56 \pm 3,04$ \\
\cline { 2 - 6 } & 9 & $7,98 \pm 0,20$ & $20,23 \pm 0,57$ & $43,15 \pm 1,82$ & $82,78 \pm 3,51$ \\
\hline \multirow{4}{*}{ II } & 12 & $8,07 \pm 0,24$ & $20,90 \pm 0,98$ & $43,38 \pm 1,37$ & $83,41 \pm 2,37$ \\
\cline { 2 - 6 } & 18 & $7,99 \pm 0,25$ & $20,76 \pm 1,32$ & $43,19 \pm 1,81$ & $82,78 \pm 3,58$ \\
\cline { 2 - 6 } & 0 & $8,02 \pm 0,24$ & $20,25 \pm 0,79$ & $43,62 \pm 1,44$ & $82,99 \pm 2,73$ \\
\cline { 2 - 6 } & 1 & $7,87 \pm 0,25$ & $21,74 \pm 0,97$ & $44,08 \pm 1,56$ & $84,19 \pm 2,89$ \\
\cline { 2 - 6 } & 6 & $9,03 \pm 0,25$ & $22,93 \pm 1,12$ & $45,07 \pm 2,04$ & $82,91 \pm 2,63$ \\
\cline { 2 - 6 } & 9 & $9,13 \pm 0,32$ & $18,12 \pm 0,80$ & $38,37 \pm 1,39$ & $83,38 \pm 3,63$ \\
\hline \multirow{4}{*}{ III } & 12 & $10,05 \pm 0,39$ & $23,14 \pm 1,07$ & $45,39 \pm 1,84$ & $85,02 \pm 4,16$ \\
\cline { 2 - 6 } & 18 & $11,02 \pm 0,34$ & $24,74 \pm \pm 0,94$ & $47,07 \pm 2,01$ & $87,29 \pm 3,08$ \\
\cline { 2 - 6 } & 0 & $8,06 \pm 0,26$ & $20,18 \pm 1,05$ & $43,91 \pm 0,99$ & $82,75 \pm 2,13$ \\
\cline { 2 - 6 } & 1 & $8,37 \pm 0,33$ & $21,50 \pm 1,07$ & $44,73 \pm 1,77$ & $84,84 \pm 3,33$ \\
\cline { 2 - 6 } & 3 & $8,95 \pm 0,22$ & $22,17 \pm 0,91$ & $45,07 \pm 1,33$ & $85,20 \pm 3,51$ \\
\cline { 2 - 6 } & 9 & $9,55 \pm 0,18$ & $22,41 \pm 1,07$ & $45,73 \pm 1,52$ & $85,82 \pm 3,35$ \\
\cline { 2 - 6 } & 12 & $10,09 \pm 0,20$ & $23,27 \pm 1,07$ & $46,32 \pm 1,76$ & $86,23 \pm 3,96$ \\
\hline & 18 & $10,88 \pm 0,24$ & $23,61 \pm 1,17$ & $47,62 \pm 1,47$ & $87,06 \pm 3,53$ \\
\hline
\end{tabular}

Bảng 5. Độ hoà tan của viên nang VER.HCl 120mg giải phóng kéo dài bảo quản ơ điều kiện lão hoá cấp tốc $\left(40 \pm 2^{\circ} \mathrm{C}, 75 \pm 5 \% R H\right)(n=6 ; \bar{x} \pm S D)$

\begin{tabular}{|c|c|c|c|c|c|}
\hline \multirow{2}{*}{ Lô } & \multirow{2}{*}{ Tháng } & \multicolumn{3}{|c|}{ Tỷ lệ \% VER. HCl giải phóng theo thời gian (giờ) } \\
\cline { 3 - 6 } & & 1 & 2 & 4 & 8 \\
\hline I & 0 & $7,96 \pm 0,19$ & $20,03 \pm 0,99$ & $43,39 \pm 1,61$ & $82,05 \pm 2,98$ \\
\hline
\end{tabular}




\begin{tabular}{|c|c|c|c|c|c|}
\hline & 3 & $9,12 \pm 0,20$ & $22,80 \pm 1,11$ & $46,58 \pm 1,55$ & $86,18 \pm 2,89$ \\
\cline { 2 - 6 } & 6 & $10,52 \pm 0,49$ & $23,59 \pm 1,09$ & $46,35 \pm 1,28$ & $86,06 \pm 3,50$ \\
\hline \multirow{3}{*}{ II } & 0 & $8,02 \pm 0,24$ & $20,25 \pm 0,79$ & $43,62 \pm 1,44$ & $82,99 \pm 2,73$ \\
\cline { 2 - 6 } & 3 & $9,95 \pm 0,45$ & $23,61 \pm 1,16$ & $46,09 \pm 2,10$ & $83,21 \pm 3,41$ \\
\hline \multirow{3}{*}{ III } & 6 & $10,60 \pm 0,20$ & $20,86 \pm 1,03$ & $46,95 \pm 2,11$ & $84,95 \pm 3,68$ \\
\cline { 2 - 6 } & 0 & $8,06 \pm 0,26$ & $20,18 \pm 1,05$ & $43,91 \pm 0,99$ & $82,75 \pm 2,13$ \\
\cline { 2 - 6 } & 3 & $9,56 \pm 0,23$ & $22,94 \pm 0,99$ & $46,32 \pm 1,26$ & $86,19 \pm 3,19$ \\
\hline
\end{tabular}

Nhận xét: Sau 18 tháng theo dõi độ ổn định ở điều kiện thực và 6 tháng ở điều kiện lão hoá cấp tốc, độ hòa tan của viên nang VER.HCl 120 mg giải phóng kéo dài thay đổi không đáng kể và vẫn đạt các yêu câu của USP 41.

\section{KẾT LUẬN}

Đã đánh giá được độ ổn định của chễ phẩm khi bảo quản 18 tháng ở điều kiện thực và 6 tháng ở điều kiện lão hóa cấp tốc, các chỉ tiêu chất lượng của viên nang VER.HCl $120 \mathrm{mg}$ giải phóng kéo dài (hình thức viên, hàm lượng, độ hòa tan) thay đổi không đáng kể và đạt yêu cầu tiêu chuẩn cơ sở của chế phẩm.

\section{TÀl LIỆ THAM KHẢO}

1. Bộ Y tế (2009), Dược thư quốc gia việt nam, Nhà xuất bản Y Học, tr. 979 - 981.

2. Martindale The Complete Drug Reference (2014), Thirty-eighth Edition, Pharmaceutical
Press, pp. 1522-1526.

3. BNF 73 (2017), Monograph: Verapamil hydrochloride, Pharmaceutical Press, London, pp. 155-156.

4. Truong Duc Manh, Vo Xuan Minh, Phan Thi Hoa, Nguyen Van Bach, Dinh Dinh Chinh (2021), Research on film coating formulation of sustained release pellets of verapamil hydrochloride, Tạp chí Y Dược học Quân sự, số 4, tr. $134-145$.

5. Food and drug Administration- USA (2003), Guidance for Industry: Q1A (R2) Stability Testing of New Drug Substances and Products.

6. ASEAN (2005), Guideline on Stability Study of Drug Product.

7. USP 41- NF 36 CD (2018), Monograph: verapamil hydrochloride extended-release tablets.

\section{KHẢO SÁT SỰ HÀI LÒNG CỦA THÀNH VIÊN GIA ĐÌNH NGƯờI BÊNH ĐỐI VỚI NHÂN VIÊN Y TẾ THUộC BỆNH VIỆN BỆNH NHIỆT ĐỚI HẢI DƯO'NG NĂM 2019}

\begin{abstract}
Phạm Thị Nhuyên***, Trần
\end{abstract}

\section{TÓM TẮT}

Mục tiêu: Đánh giá sự hài lòng của thành viên gia đình người bệnh (TVGĐ NB) với bác sĩ (BS), điêuu dưỡng (ĐD) và đối với các yếu tố khác thuộc Bệnh viện bệnh Nhiệt đới Hải Dương năm 2019. Đối tượng và phương pháp: Nghiên cứu mô tả cắt ngang 72 thành viên gia đình người bệnh điều trị tại Bệnh viện Nhiê̂t đới Hải Dương năm 2019. Kết qủa và kết luận: Mức độ hài lòng của TVGĐ NB với BS tại Bệnh viện bệnh Nhiệt đới Hải Dương: khám, giải thích về

*Trường Đại học KH và CN Hà Nội

**Trường Đại học Y tê Công cộng

***Trướng Đại học Thành Đông

Chịu trách nhiệm chính: Phạm Thị Nhuyên

Email: mhuyenmd@yahoo.com.vn

Ngày nhận bài: 2/10/2021

Ngày phản biện khoa học: 29/10/2021

Ngày duyệt bài: 12/11/2021
Nguyễn Thu Hà*, Trần Trọng Hải**, hư Nguyên***, Phí Thị Nguyệt Thanh***, Nguyễn Đức Sơn $* * *$, Nguyễn Thu Hà $* * *$ tình trạng bệnh, chẩn đoán, trực tiếp điều trị là 98,57\%. Mức độ hài lòng của TVGĐ NB với ĐD tai Bệnh viện bệnh Nhiệt đới Hải Dương, gồm: phổ biến những thông tin cân thiết khi vào Bệnh viện và trong quá trình điểu trị $(100 \%)$; Có lời nói, cử chỉ, thái độ thân thiện với người bệnh (NB) và TVGĐ NB $(98,61 \%) ;$ Tư vấn chế độ chăm sóc NB là $(100 \%)$; Tận tình giúp đỡ NB, TVGĐ̈, không gợi ý, đòi tiền quà̀ $(97,22 \%)$; Công khai thuốc $(95,83 \%)$ và hướng dẫn sử dụng thuốc (100\%). Mức độ hài lòng của TVGĐ NB với các yếu tố khác của Bệnh viện bệnh Nhiệt đới Hải Dương: Thủ tục hành chính nhanh gọn (100\%); Vệ sinh sạch sẽ $(94,45 \%)$; Cơ sở hạ tâng và trang thiết bị hiện đại $(65,28 \%)$.

Tư khóa: Sự hài lòng, thành viên gia đình, người bệnh, bác sĩ, điều dưỡng, bệnh nhiệt đới.

\section{SUMMARY \\ SATISFACTION SURVEY OF PATIENT \\ FAMILY MEMBERS AT HAI DUONG TROPICAL HOSPITAL IN 2019}

